\title{
Stain Normalization using Sparse AutoEncoders (StaNoSA): Application to digital pathology
}

\author{
Andrew Janowczyk ${ }^{1}$, Ajay Basavanhally ${ }^{2}$, Anant Madabhushi ${ }^{1}$ \\ ${ }^{1}$ Case Western Reserve University, Cleveland, Ohio \\ ${ }^{2}$ Inspirata, Inc., Tampa, Florida
}

\begin{abstract}
Digital histopathology slides have many sources of variance, and while pathologists typically do not struggle with them, computer aided diagnostic algorithms can perform erratically. This manuscript presents Stain Normalization using Sparse AutoEncoders (StaNoSA) for use in standardizing the color distributions of a test image to that of a single template image. We show how sparse autoencoders can be leveraged to partition images into tissue sub-types, so that color standardization for each can be performed independently. StaNoSA was validated on three experiments and compared against five other color standardization approaches and shown to have either comparable or superior results.

Keywords: digital histopathology, stain normalization, deep learning, image processing
\end{abstract}

\section{Introduction}

Digital pathology (DP) is the process by which histology slides are digitized to produce high resolution images via whole slide digital scanners [1. These digitized slides afford the possibility of applying image analysis techniques to tissue images for the purpose of object detection, segmentation, and tissue classification. These automated image analysis algorithms are very relevant for nuclei detection, mitosis quantification, and tubule counting. Additionally these image analysis algorithms provide the ability of performing higher level, supervised learning tasks such as disease grading, thereby enabling the development 
of decision support algorithms for pathologists [2].

Prior to evaluation of the specimen under the microscope by a pathologist, the tissue is invariably treated by artificial or natural agents, to stain the various cellular structures. Hemotoxylin and Eosin $(\mathrm{H} \& \mathrm{E})$ is one of the the most routinely used stains for evaluating disease morphology, an example of which is shown in Figure 1. The hemotoxylin provides a blue or purple appearance to the nuclei while the eosin renders eosinophilic structures (e.g., cytoplasm, collagen, and muscle fibers) a pinkish hue.

Since the staining process is a chemical one, there are many variables which can drastically change the overall appearance of the same tissue. For example, the specimen thickness, concentration of the stain, manufacturer, time, and temperature at which the stain is applied can have significant implications for the appearance of the final tissue specimen. Figure 1 , shows an H\&E stained gastrointestinal (GI) biopsy tissue image. Figure $1 \mathrm{~b}$ shows a sample taken from the same specimen but stained using a slightly different protocol (i.e. a different concentration of H\&E respectively), and as such, appears significantly darker.

The staining process is not the only source of visual variability in imaging of tissue specimens. The digitization process also could potentially induce changes and variability in tissue image appearance. For example Figure 1 shows the same physical specimen scanned using two different scanners. Differences in the scanning platform (e.g., bulbs, ambient illumination, sensor chips), image stitching algorithms, and acquisition technologies (e.g., compression, tiling, whiteness correction) can also induce substantive differences in appearance of the resulting tissue image.

Pathologists are specifically trained to be able to cope with these variations and for the most part do not struggle with diagnostic decision making or object counting/quantification (e.g., nuclear or mitosis counting). On the other hand, image analysis methods, especially supervised learning/classification algorithms for nuclei segmentation or tissue partitioning, typically find it more difficult to cope with variations in image appearance and staining [3. For instance if an algorithm is trained to identify nuclei based off chromatic cues from a singe site, 
the variations in staining might cause the algorithm to have a large number of errors for slightly differently stained images from a different site. This is further compounded when we consider extremely large datasets that are curated from many different sites, such as The Cancer Genome Atlas (TCGA).

These variations in stain and tissue appearance have spurred recent research in development of color standardization and normalization algorithms to help improve performance of subsequent image analysis algorithms [3, 4]. Often, this occurs by identifying a single image with the most optimal tissue staining and visual appearance, and designating this image as the "template". Subsequently all other images to be standardized have their intensity distributions mapped to match the distribution of the template image. Previous works [5, 6, 7] have suggested that partitioning the image into constitute tissue subtypes (i.e., epithelium, nuclei, stroma, etc.) and attempting to match distributions on a tissue-per-tissue basis is more optimal compared to an approach which involves simply aligning global image distributions between the target and template images. In the context of histopathology this process might involve first identifying stromal tissue, nuclei, lymphocytes, fatty adipose tissue, cancer epithelium both within the target and template images and then specifically establishing correspondences between the tissue partitions in the two images. Subsequently the tissue specific distributions could then be aligned between the target and template images. While these tissue specific alignment procedures [8] have had more success compared to global intensity alignment approaches [9], successfully identifying the partitions remains an open challenge. For example, nuclei segmentation on its own is a large area of research [10, 11, 12, 13, yet represents only a single histologic primitive. It is therefore clear that more powerful and flexible approaches are needed for automated partitioning of the entire tissue image into distinct tissue compartments.

Our approach, Stain Normalization using Sparse AutoEncoders (StaNoSA), is based off the intuition that similar tissue types will be clustered close to each other in a learned feature space. This feature space is derived in an unsupervised manner, releasing it from the requirement of domain specific knowledge such as 
having to know the "true" color of the tissue stains, a requirement for a number of other approaches [14. Our approach employs sparse-auto encoders (SAE), a type of deep learning approach which through an iterative process learns filters which can optimally reconstruct an image. These filters provide the feature space for our approach to operate in. Once the pixels are appropriately clustered in this deep learned feature space into their individual tissue sub-types, tissue distribution matching (TDM) can occur on a per channel, per cluster basis. This TDM step allows for altering the target image to match the template image's color space.

The main contribution of this work is a new TDM based algorithm for color standardization for digital pathology images and which employs sparse autoencoders for automated tissue partitioning and establishing tissue specific correspondences between the target and template images. Autoencoding is the unsupervised process of learning filters which can most accurately reconstruct input data when transmitted through a compression medium. By performing this procedure as a multiple layer architecture, increasingly sophisticated data abstractions can be learned [15]. Additionally as part of our approach we perturb the input data with noise and attempt to recover the original unperturbed signal, an approach termed denoising auto-encoders [15, that has been shown to yield robust features. StaNoSA is thus a fully automated way of transforming images of the same stain type to the same color space so that the amount of variance from (a) technicians, (b) protocols, and (c) equipment could be minimized.

The rest of the paper is organized as follows. Section 2 involves a review of the previous work in the field. Section 3 describes the approach (StaNoSA) and associated algorithms. Section 4 rigorously evaluates the method across 2 different datasets and compares the approach with a state of the art approach and 4 other common methods. Section 5 contains the discussion. Finally, in Section 6 we present our concluding remarks. 


\section{Previous Work and Novel Contributions}

Previous approaches $[8]$ to color normalization for digital histopathology images tend to fall into one of two categories. The first set of approaches exploit staining characteristics directly, such as Beer-Lambert's law through color deconvolution [16. They attempt to divide the image color space into individual stain contributions and normalize these individually. The second category of algorithms take a statistical approach which rely on finding clusters of pixels belonging to a similar tissue-types (e.g., nuclei, epithelium, stroma). Once these partitions are identified, and correspondences across different images is established, the color distributions can be matched to a template distribution, so that each histologic entity can be manipulated separately. While a thorough review of the state of the art is out of the scope of this paper, below, we briefly discuss and differentiate approaches most relevant to StaNoSA. Specifically, while many previous works operate solely in the gray color space [17, 18, 19, we limit our discussion primarily to color based approaches since the specific application considered in this work is digital pathology (and specifically H\&E stained imagery). We do however direct the interested reader to a very recent review of state of the art $[8$ approaches for color normalization.

\subsection{Stain Specific Algorithms}

The most popular and well cited backbone for stain specific normalization algorithms is color deconvolution (CD) [16. CD is based on the idea that the individual stains can be separated and subsequently normalized independently. Subsequently, the normalized color channels can be recombined to recreate an improved version of the original color image. The underlying stain signals are identified and separated by taking advantage of the Beer-Lambert's law [16] which is then used to linearly estimate the contribution of each stain to the final pixel color. The stain matrix describes, through coefficients for each stain, how much each stain contributes to the final pixel color value. This approach has been shown to work well when the color matrix can be reliably and definitively determined [16]. 
On the other hand, identifying this matrix requires either (a) images which are scanned on the same scanner using only a single stain to compute the deconvolution matrix (a type of color calibration approach) or (b) a semi-supervised approach wherein users are required to select pixels corresponding to the relevant classes as shown in [20].

While in [16] the authors were able to obtain accurate estimations of the stain matrix on their specific dataset, the same stain matrix may not adequately generalize to images from other sites and scanning platforms. They describe how ideal matrices can be estimated but the process requires the usage of control tissue and a single stain scanning process which may not always be possible. It may also not be possible to identify a stain matrix that simultaneously addresses variations due to scanning platforms. Magee et al. in [21] discussed this limitation as well by alluding to the difficulty in identifying the appropriate parameters for the deconvolution matrix.

\subsection{Clustering Type Algorithms}

A second category of approaches aims to identify distinct tissue clusters in a feature space and aims to perform a cluster-to-cluster distribution alignment across the template and target images. Some approaches have attempted to decompose an image using non-negative matrix factorization [22, only requiring knowledge of the number of stains used. Other approaches aim to classify every pixel in the image to one of multiple different tissue classes. In 23] the Expectation Maximization approach was employed to create a fuzzy labeling of the image into distinct tissue classes. However, these approaches [8] may not work optimally if the individual tissue clusters are not proportionately represented in the target and template images. For example, the EM approach in 23] could end up mapping similar appearing regions from distinct tissue classes to the same cluster. During the the TDM process this can lead to distorted color density distributions. 


\subsection{Novel Contributions}

In this paper we present a novel technique, StaNoSA, for fully unsupervised normalization of images to a template image. StaNoSA solely requires as input a template image, as opposed to domain specific information such as mixing coefficients or stain properties. This approach therefore enables the ability to shift a target image in the color domain to more accurately resemble the template image. We extend the approach initially presented in [23] by considering not solely chromatic value of pixels for clustering. Instead, we employ a fully unsupervised deep learned bank of filters. These filters represent optimal [24] filters for image reconstruction via compression. By operating in this filtered space, we obtain significantly more robust pixel classes. These classes are not tightly coupled to individual stain classes, and thus the approach has a greater likelihood of success in the case of challenging images, images where one might find new tissue classes or a disproportionate tissue distribution compared to the template image.

A representative result showing the application of StaNoSA is presented in Figure 1. where we normalized the target image (Figure 1b) into the color space of the template image (Figure 1 1 ) to produce a result (Figure 1 has very similar color characteristics as the template color space. This process was applied by clustering the pixels in a sparse auto-encoded feature space so that respective tissue partitions could be aligned using their respective color distributions. By using individual tissue partitions, StaNoSA is able to more sensitively modify the color space as compared to a global method where all pixels are considered concurrently. We can see the resulting density functions of the pixels in 3 channel RGB, from which we can see that the target image's distribution (Figure 1e) is heavily skewed towards the left. After normalization (Figure 1:) the probability distribution of the 3 channels more closely resembles that of the template image (Figure 1 $\mathrm{d}$ ).

The primary novel contributions of this work may be summarized as follows.

- StaNoSA is a domain agnostic approach, in that it does not rely on specif- 
ically knowing stain values or tissue values allowing the user to choose the template to standardize to.

- StaNoSA leverages a sparse auto-encoder (SAE) [15, 25] as the core method to automatically partition the image into distinct tissue categories. DL methods have been shown to be robust and accurate for image partitioning 14.

- First of its kind study in which we quantitatively measure intra-scanner and inter-scanner variances of digitized $\mathrm{H} \& \mathrm{E}$ histology images.

\section{Methods}

\subsection{Notation}

For all methods, we define the dataset $\mathbf{Z}=\left\{\mathcal{C}_{1}, \mathcal{C}_{2}, \ldots \mathcal{C}_{\mathcal{M}}\right\}$ of $\mathcal{M}$ images, where an image $\mathcal{C}=(C, \psi)$ is a $2 \mathrm{D}$ set of pixels $c \in C$ and $\psi$ is the associated vectorial function which assigns $\mathrm{RGB}$ values. $\mathcal{T}=\mathcal{C}_{a} \in \mathbf{Z}$ is chosen from $\mathbf{Z}$ as the template image by which all other images in the dataset will be normalized to. Without loss of generality we chose $\mathcal{S}=\mathcal{C}_{b} \in \mathbf{Z}$ to be the "target image", which is to be normalized into the color space of $\mathcal{T}$. See Table 1 for additional notation used in this manuscript.

\subsection{Deep Learning of Filters from Image Patches}

Denoising auto-encoders are leveraged in this work to learn representative feature spaces for optimizing tissue partitioning. We present them briefly below.

\subsubsection{One Layer Autoencoder}

From $\mathcal{T}$ we randomly select $p \in \mathbb{R}^{v \times v \times 3}$ sub-images, or patches, of $v \times v$ dimension in 3-tuple color space (RGB) (see Figure 3). We set $V=v \times v \times 3$ to simplify notation. These values are reshaped into a data matrix $\mathbf{X} \in \mathbb{R}^{p \times V}$ of $\mathbf{x} \in \mathbb{R}^{1 \times V}$ samples. This matrix $\mathbf{X}$ forms the basis from which the filters will be learned. 
A simple one layer auto-encoder can be defined as having both an encoding and decoding function. The encoding function encodes a data sample from its original dataspace of size $V$ to a space of size $h$. Consequently, the decoding function decodes a sample from $h$ space back to $V$ space.

We use notation from [15] where they show a typical encoding function for a sample $\mathbf{x}$ is

$$
\mathbf{y}=f_{\theta}(\mathbf{x})=s(\mathbf{W} \mathbf{x}+\mathbf{b}),
$$

parameterized by $\theta=\{\mathbf{W}, \mathbf{b}\}$. $\mathbf{W}$ is a $h \times V$ weight matrix, $\mathbf{b} \in \mathbb{R}^{1, V}$ is a bias vector, and $s$ is an activation function (in this work $s$ is assumed to be the hyperbolic tangent function). The reconstruction of $\mathbf{x}$, termed $\mathbf{z}$, proceeds similarly using a decoding function $\mathbf{z}=g_{\theta^{\prime}}(\mathbf{y})=s\left(\mathbf{W}^{\prime} \mathbf{y}+\mathbf{b}^{\prime}\right)$ with $\theta^{\prime}=\left\{\mathbf{W}^{\prime}, \mathbf{b}^{\prime}\right\}$. Here $\mathbf{W}^{\prime}$ is a $V \times h$ weight matrix, and $\mathbf{b}^{\prime} \in \mathbb{R}^{1, h}$ is a bias vector.

We use stochastic gradient descent [26] to optimize both $\theta$ and $\theta^{\prime}$ relative to the average reconstruction error, $\theta^{*}$. To further use notation from [15], this error is defined as:

$$
\theta^{\star}, \theta^{\prime \star}=\arg \min _{\theta, \theta^{\prime}} \frac{1}{p} \sum_{i=1}^{p} L\left(\mathbf{x}^{(i)}, \mathbf{z}^{(i)}\right)
$$

where the loss function $L$ is a simple squared error $L(\mathbf{x}, \mathbf{z})=\|\mathbf{x}-\mathbf{z}\|^{2}$.

\subsubsection{Expansion to Multiple Layers and Denoising}

It has been shown that by applying these auto-encoders in a greedy layer wise fashion, higher level abstractions, in a lower dimensional space, may be learned. In particular, this means taking the output from layer $l$, i.e., $\mathbf{y}^{(l)}$, and directly using that as the input $\left(\mathbf{x}^{(l+1)}\right)$ at the next layer to learn a further abstracted output $\mathbf{y}^{(l+1)}$ by re-applying Equation 2, Layer 1 has input $\mathbf{x}^{(1)}$ of size $\mathbb{R}^{1 \times V}$ and output $\mathbf{y}^{(1)}$ of size $\mathbb{R}^{1 \times h^{(1)}}$. Layer 2 thus has $\mathbf{x}^{(2)}=\mathbf{y}^{(1)}$ of size $\mathbb{R}^{1 \times h^{(1)}}$ and output $\mathbf{y}^{(2)}$ of size $\mathbb{R}^{1 \times h^{(2)}}$. This layering can continue as deemed necessary.

Additionally, by intentionally adding noise to the input values of $\mathbf{X}$, more robust features across all levels may be learned. Briefly, we formalize this by 
saying $\hat{\mathbf{X}}=\epsilon(\mathbf{X})$, where $\epsilon$ is a binomial corrupter which sets elements in $\mathbf{X}$ to 0 with probability $\phi$. Using $\hat{\mathbf{x}}$ in place of $\mathbf{x}$ in Equation 1, results in the creation of a noisy lower dimensional version $\hat{\mathbf{z}}$. This reconstruction is then used in Equation 2 in place of $\mathbf{z}$, while the original $\mathbf{x}$ remains in place.

\subsubsection{Generating feature space representations for image}

Once the filters are learned for all levels, we apply the full hierarchy of encoders on both the template image, $\mathcal{T}$, and a "moving image", $\mathcal{S}$. The main assumption of our approach is that regardless of visual appearance, pixels belonging to the same tissue class will have similar responses to the learned filters. Figure 4 shows an example of this procedure using two images of the same tissue stained, but with different stain concentrations. It can be seen that although the visual appearance of these two images is quite different, the filters appear to elicit a similar response to similar tissue classes in the image. As such, this feature space allows for an unsupervised clustering process to identify tissue partitions. In Figure 4(c) and 4(f), each unique grayscale value represents a different cluster, suggesting that pixels of similar textural appearance tend to be grouped into the same cluster. However we note that there is no obvious connection between the individual clusters and the tissue classes present in the image. This therefore suggests that we need a subsequent unsupervised clustering step in order to align the tissue partitions.

\subsection{Unsupervised Clustering}

Once we obtain the filter responses for $\mathcal{T}$ and $\mathcal{S}$, i.e., $\dot{\mathcal{T}}$ and $\dot{\mathcal{S}}$ respectively, we aim to cluster them into individual partitions. To this end, we employ a standard $k$-means approach on $\mathcal{T}$ to identify $K$ cluster centers. Subsequently, we assign each of the pixels in $\dot{\mathcal{S}}$ to its nearest cluster, without performing any updating. This process yields $\mathcal{S}$, a cluster indicator variable. This approach helps to assuage some of the typical instability issues commonly associated with $k$-means. We note that although the clustering is taking place on a pixel level, the feature space has been computed on a 32 × 32 window around each pixel, 


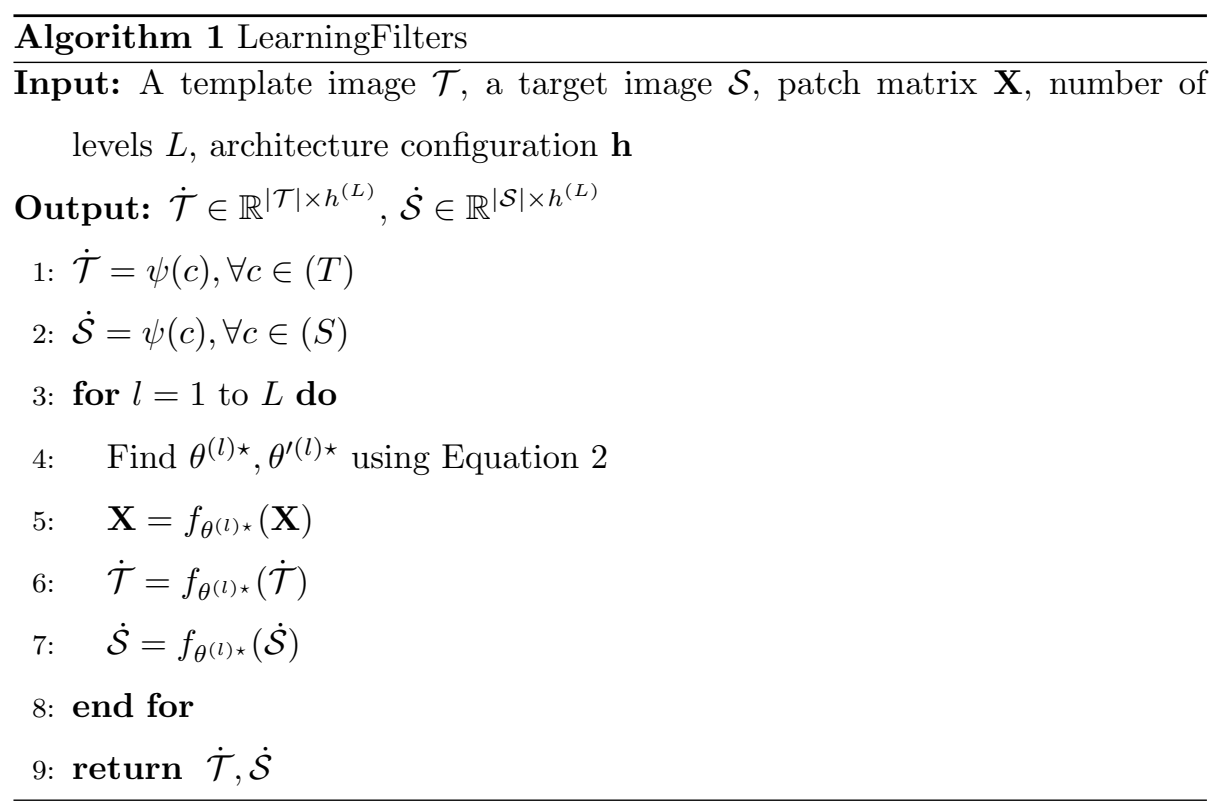

providing the necessary context for our clusters to be better informed.

In [23], for instance, the $K$ clusters loosely corresponded to individual tissue classes such as nuclei, stroma or lymphocytes. However, the maximum $K$ that could be chosen in 23. was implicitly limited. In the case of StaNoSA, we use a much larger $K$, on the order of 50. A larger number of clusters potentially allows for more precisely tuned clusters.

\subsection{Histogram Shifting}

Once the clusters are defined, a more precise color standardization process can take place. For each $K$, operating on a per channel basis, we standardize the distribution of the moving images to that of the template images 9 . We present this approach in Algorithm 2 which is the basis for the implementation of the imhistmatch function in Matlab.

As was mentioned in Section 2, a common problem with global normalization techniques is the inability to account for both tissue class proportions and in cases where the color distributions are already similar, StaNoSA is able to minimize the overall error (see Experiment 1 in Section 4). By assigning the 


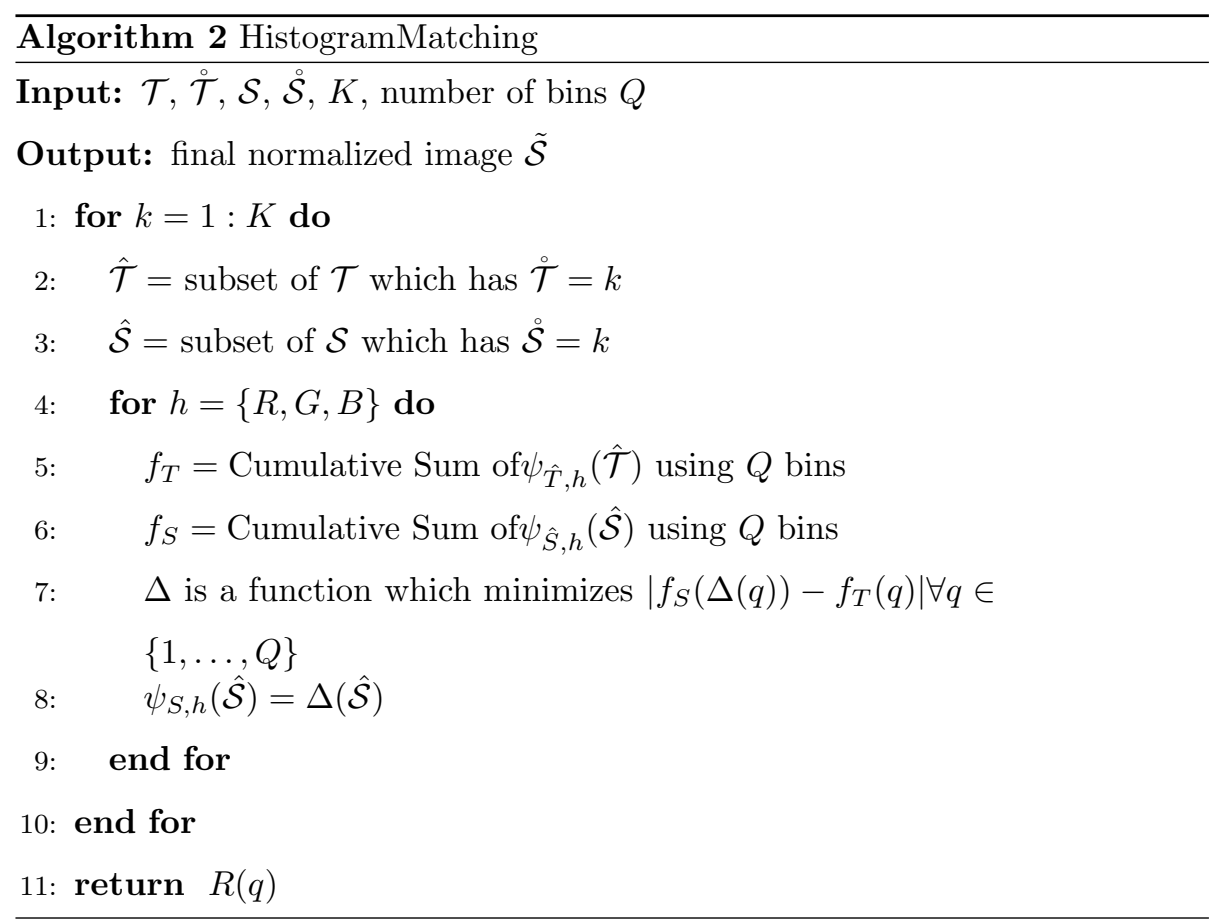

pixels in $\mathcal{S}$ to a larger number of clusters, but not performing updating, the disproportional class representation is effectively managed.

\section{Experimental Evaluation}

To rigorously evaluate our approach, we perform three experiments, each focused on directly addressing a different reason for variance in color and appearance of pathologic tissue slides. Specifically we attempted to address variations induced by (a) differences in platform and scanners, and (b) staining. Additionally we also evaluated the performance of a nuclear detection algorithm on the pre- and post-standardized images to evaluate the role of color standardization methods in facilitating object detection. Using three different datasets, as shown in Table 2, we attempted to evaluate StaNoSA when (a) different concentrations of $\mathrm{H} \& \mathrm{E}$ were deliberately varied in the same tissue section and (b) when the same slide was scanned multiple times on two different platforms. Additionally we also compare our approach to 5 other color normalization approaches. 


\begin{tabular}{c|c|c|c|l} 
Name & Organ & Stain & Images & \multicolumn{1}{|c}{ Importance } \\
\hline$S_{1}$ & Breast & HE & 25 at $40 \mathrm{x}$ & $\begin{array}{l}\text { Same slides scanned on different } \\
\text { equipment } \\
S_{2}\end{array}$ \\
GI & HE & 175 at $40 \mathrm{x}$ & $\begin{array}{l}\text { Adjacent slices stained using dif- } \\
\text { ferent protocols } \\
\text { A subset of } S_{2} \text { containing manual } \\
\text { annotations of nuclei boundaries }\end{array}$
\end{tabular}

Table 2: The three unique datasets used in the evaluation of Stanosa

\subsection{Datasets}

\subsubsection{Dual Scanner Breast Biopsies}

The $S_{1}$ dataset consists of 5 breast biopsy slides. Each slide was scanned at 40x magnification 3 times on a Ventana whole slide scanner and once on a Leica whole slide scanner, resulting in 20 images of dimension 100,000 x 100,000 pixels. Each set of 4 images (i.e., 3 Ventana and 1 Leica), were mutually co-registered so that from each biopsy set, 10 sub-regions of 1,000 x 1,000 could be extracted. This resulted in 200 images: 10 sub-images from 4 scans across 5 slides. The slides were formalin fixed paraffin embedded and stained with Hematoxylin and Eosin $(H \& E)$, each slide having some evidence of cancer presence. Since the subimages are all produced from the same tissue slide, this allows for a rigorous examination of intra- and inter-scanner variation.

\subsubsection{Gastro-Intestinal Biopsies of differing protocols}

The $S_{2}$ dataset consists of slices taken from a single cancer positive Gastro Intestinal (GI) biopsy. The specimen was formalin fixed paraffin embedded and had 7 adjacent slices sectioned from the same biopsy tissue and subjected to different staining protocols: $\mathrm{HE}, \mathrm{H} \downarrow \mathrm{E}, \mathrm{H} \uparrow \mathrm{E}, \downarrow \mathrm{HE}, \downarrow \mathrm{H} \downarrow \mathrm{E}, \uparrow \mathrm{HE}$, and $\uparrow \mathrm{H} \uparrow \mathrm{E}$, where $\uparrow$ and $\downarrow$ indicate over- and under-staining of the specified dye. These intentional staining differences were intended to simulate the typical variability seen in clinical settings, especially across different sites. Each slide was then digitized using an Aperio whole-slide scanner at 40x magnification $(0.25 \mu \mathrm{m}$ per 
pixel), from which 25 random 1,000 x 1,000 resolution images were cropped at 20x magnification. Representative images within $S_{2}$ can be seen in Figure 6 .

\subsubsection{Gastro-Intestinal Biopsies of differing protocols with annotations}

The $S_{3}$ dataset is a subset of $S_{2}$ manual annotations of the nuclei. From each of the 7 different protocols, as discussed above, a single image patch of about $1,000 \times 1,000$ pixels was cropped at 40x magnification. Nuclear boundaries were then delineated by an expert pathologist.

\subsection{Comparative Color Standardization Schemes}

\subsubsection{Normalization}

The parameters used for each experiment associated with our approach (StaNoSA) are as follows: 250,000 patches of size $(v) 32 \times 32$, were extracted from only template image. A 2 layer SAE was created with the first layer containing 100 hidden nodes $\left(h_{1}\right)$ and the second layer ten $\left(h_{2}\right)$. The denoising variable was set to $\epsilon=.2$. Histogram equalization took place using $Q=128$ bins. Additionally, the following pre-processing steps were applied to each cohort of images: ZCA whitening and global contrast normalization [27.

\subsubsection{Direct}

This "approach" only employs the raw image without any modifications to quantify what would happen if no normalization was undertaken at all.

\subsubsection{Global Standardization}

This approach is similar to Algorithm 2, except assuming that $K=1$, in which all pixels in the image belong to a single cluster. Again, $Q=128$ bins were used for the histogram matching process.

\subsubsection{Four Additional Approaches}

We also compared StaNoSA against the publicly available stain normalization toolbox presented in [8]. This toolbox also comprises four additional approaches, Reinhard et al. (RH) [20, Macenko et al. (MM) 28], Histogram 
Specification (HS) 9], and Khan et al. (DM) 8]. We direct the reader to their respective papers for implementation details.

\subsection{Implementation Details}

It took 5 hours to train the deep learning network using a Nvidia M2090 GPU with 512 cores at $1.3 \mathrm{ghz}$ and under 3 minutes to generate each output image needed for the clustering mechanism. Subsequently, for images of size $1,000 \times 1,000$, the entire clustering and shifting process takes under 1 minute on a 4 core $2.5 \mathrm{ghz}$ laptop computer. All deep learning was developed and performed using the popular open source library Pylearn2 29] which uses Theano 30] for its backend graph computation.

\subsection{Experiment 1: Standardization across scanners \\ 4.4.1. Design}

We aim to evaluate the extent of differences between colors for the same slide scanned multiple times on the same scanner $\left(C_{j, i}^{i n}\right.$, where $i \in\{1,2,3\}$ represents the scan number and $j \in\{1, \ldots, N\}$ represents the image number) to compute intra-scanner error $\left.\left(\mu_{i, k}^{i n}=\sum_{j} S S D\left(C_{j, 1}^{i n}, C_{j, 2}^{i n}\right) / N, i \in\{1,2,3\}, k \in\{1,2,3\}\right)\right)$ and the error in scanning the same slide across a different platform $\left(C_{j}^{i t}, j \in\right.$ $\{1, \ldots, N\})$ to compute the inter-scanner error $\left(\mu_{i}^{i t}=\sum_{j} S S D\left(C_{j, i}^{i n}, C_{j, 1}^{i t}\right) / N, i \in\right.$ $\{1,2,3\}))$. The SSD error involves taking each color channel and computing a 128 bin histogram and use the sum of the squared difference of the bins for each of the color channels. The optimal error would of course be 0 if both images were identical.

Our aim in this experiment was to evaluate whether StaNoSA could help bring $\mu^{i t}$ into $\mu^{i n}$ range. More specifically the goal was to assess whether $\mid \mu^{i t}-$ $\mu^{i n} \mid<\gamma$ where $\gamma$ is a predefined threshold that represents $\sigma^{i n}$, typical intrascannar variance. While ideally we would have liked to perform a pixel level mean squared error difference between a template and target image, this was not possible on account of two issues. Firstly, the image resolution for slides digitized on the scanners is not identical. This in turn would actually require 
image re-scaling and interpolation which in turn would likely induce additional error. Secondly, there are visible tiling artifacts visible on both the intra/inter scanner images, making the pixel level error nearly impossible to determine. Instead, we use the SSD measure (introduced above) as a surrogate.

Using the 200 images from dataset $S_{1}$, we perform two experiments. First, we compute the mean and variance SSD per image $\left(\mu^{i n}, \sigma^{i n}\right)$ across the 3 Ventana scans $\left(S_{1, V_{i}}, i \in\{1,2,3\}\right)$ and apply StaNoSA to determine if it is possible to reduce $\mu^{i t}, \sigma^{i t}$. Second, we use the co-registered Leica scan $\left(S_{1, L}\right)$ from each set and apply StaNoSA to $S_{1, V_{i}}, i \in\{1,2,3\}$, measuring $\mu^{i t}$ before and after StaNoSA is performed.

\subsubsection{Results}

After comparing errors, we note that the SSD $\mu^{i n}$ is about .03 (see Figure 5). The global normalization (GL) approach when applied to $S_{1}$ seems to exacerbate the existing error. On the other hand, StaNoSA is clearly shown to not only reduce $\mu^{i n}(.01)$ but also substantially reduce the $\sigma_{i n}$ seen within samples.

We additionally also examined if and how variations in image appearance on account of the use of different scanners could be reduced (see Figure 5). In this instance, we see that the GL technique does indeed reduce $\mu^{i t}$ from about 14 to .096, but our StaNoSA approach reduces the error down even more substantially to .047 which is on the order of $\mu^{i n}$ as shown in Figure 2(b) which has a $\mu^{i n}$ of .0473. In all cases, we can see that StaNoSA reduces both $\mu^{i n}, \mu^{i t}$ and $\sigma^{i n}, \sigma^{i t}$.

\subsection{Experiment 2: Standardization Across Stain Protocols}

\subsubsection{Design}

We aim to determine how well StaNoSA can succeed at minimizing SSD by bringing $S$ into the color space of $T$. In this experiment we use $S_{2}$ as a way to quantify how well the mean SSD error $\left(\mu_{p, q}=\sum_{i} S S D\left(C_{p}, C_{q}^{i n}\right) / N, i \in\right.$ $\{1, \ldots, N\}$, where $p, q$ represent any of the 7 stain proctols (e.g., $\mathrm{HE}, \mathrm{H} \downarrow \mathrm{E}, \mathrm{H} \uparrow \mathrm{E}$, $\downarrow \mathrm{HE}, \downarrow \mathrm{H} \downarrow \mathrm{E}, \uparrow \mathrm{HE}$, and $\uparrow \mathrm{H} \uparrow \mathrm{E})$ can be reduced across $S_{2}$. In each instance we arbitrarily select $T$ from the group and attempt to standardize the remaining 
images to that image and compute $\mu_{p, q}$ and the variance $\sigma_{p, q}$. We do this for all protocol pairings (i.e., $p, q$ ) and images: 7 protocols versus the remaining 6 with 25 images each resulting in 1,050 normalization operations. We report both $\mu_{p, q}$ and $\sigma_{p, q}$ across all protocols.

4.5.2. Results

\begin{tabular}{|c|c|c|c|c|c|c|c|c|}
\hline & $\mathrm{HE}$ & $\uparrow \mathbf{H} \uparrow \mathbf{E}$ & $\downarrow \mathbf{H} \downarrow \mathbf{E}$ & $\downarrow \mathbf{H E}$ & $\mathbf{H} \downarrow \mathbf{E}$ & $\mathbf{H} \uparrow \mathbf{E}$ & $\uparrow \mathbf{H E}$ & \\
\hline \multirow{7}{*}{ HE } & $\mathrm{N} / \mathrm{A}$ & $0.35 \pm 0.02$ & $0.43 \pm 0.03$ & $0.43 \pm 0.03$ & $0.45 \pm 0.03$ & $0.46 \pm 0.03$ & $0.54 \pm 0.03$ & Direct \\
\hline & $\mathrm{N} / \mathrm{A}$ & $0.09 \pm 0.00$ & $0.12 \pm 0.00$ & $0.12 \pm 0.00$ & $0.11 \pm 0.00$ & $0.11 \pm 0.00$ & $0.10 \pm 0.00$ & GL \\
\hline & $\mathrm{N} / \mathrm{A}$ & $0.05 \pm 0.00$ & $0.06 \pm 0.00$ & $0.05 \pm 0.00$ & $0.04 \pm 0.00$ & $0.04 \pm 0.00$ & $0.04 \pm 0.00$ & StaNoSA \\
\hline & $\mathrm{N} / \mathrm{A}$ & $0.07 \pm 0.00$ & $0.10 \pm 0.00$ & $0.10 \pm 0.00$ & $0.08 \pm 0.00$ & $0.09 \pm 0.00$ & $0.07 \pm 0.00$ & DM \\
\hline & $\mathrm{N} / \mathrm{A}$ & $0.41 \pm 0.02$ & $0.34 \pm 0.02$ & $0.37 \pm 0.02$ & $0.33 \pm 0.02$ & $0.35 \pm 0.02$ & $0.38 \pm 0.02$ & HS \\
\hline & $\mathrm{N} / \mathrm{A}$ & $0.35 \pm 0.05$ & $0.42 \pm 0.03$ & $0.42 \pm 0.03$ & $0.45 \pm 0.03$ & $0.45 \pm 0.03$ & $0.45 \pm 0.03$ & MM \\
\hline & $\mathrm{N} / \mathrm{A}$ & $0.48 \pm 0.02$ & $0.43 \pm 0.03$ & $0.43 \pm 0.03$ & $0.48 \pm 0.02$ & $0.47 \pm 0.02$ & $0.55 \pm 0.02$ & $\mathrm{RH}$ \\
\hline \multirow{7}{*}{$\uparrow \mathbf{H} \uparrow \mathbf{E}$} & $0.35 \pm 0.02$ & N/A & $0.39 \pm 0.01$ & $0.36 \pm 0.01$ & $0.29 \pm 0.01$ & $0.27 \pm 0.01$ & $0.25 \pm 0.02$ & Direct \\
\hline & $0.28 \pm 0.01$ & $\mathrm{~N} / \mathrm{A}$ & $0.14 \pm 0.00$ & $0.12 \pm 0.00$ & $0.10 \pm 0.00$ & $0.10 \pm 0.00$ & $0.10 \pm 0.00$ & GL \\
\hline & $0.17 \pm 0.00$ & $\mathrm{~N} / \mathrm{A}$ & $0.07 \pm 0.00$ & $0.07 \pm 0.00$ & $0.05 \pm 0.00$ & $0.05 \pm 0.00$ & $0.04 \pm 0.00$ & StaNoSA \\
\hline & $0.28 \pm 0.01$ & $\mathrm{~N} / \mathrm{A}$ & $0.13 \pm 0.00$ & $0.12 \pm 0.00$ & $0.10 \pm 0.00$ & $0.10 \pm 0.00$ & $0.09 \pm 0.00$ & DM \\
\hline & $0.31 \pm 0.03$ & $\mathrm{~N} / \mathrm{A}$ & $0.18 \pm 0.02$ & $0.17 \pm 0.01$ & $0.16 \pm 0.01$ & $0.15 \pm 0.01$ & $0.17 \pm 0.02$ & HS \\
\hline & $0.96 \pm 0.14$ & $\mathrm{~N} / \mathrm{A}$ & $0.22 \pm 0.01$ & $0.22 \pm 0.01$ & $0.20 \pm 0.02$ & $0.20 \pm 0.02$ & $0.21 \pm 0.02$ & MM \\
\hline & $0.31 \pm 0.01$ & $\mathrm{~N} / \mathrm{A}$ & $0.24 \pm 0.01$ & $0.24 \pm 0.01$ & $0.25 \pm 0.01$ & $0.24 \pm 0.01$ & $0.29 \pm 0.01$ & $\mathrm{RH}$ \\
\hline \multirow{7}{*}{$\downarrow \mathbf{H} \downarrow \mathbf{E}$} & $0.43 \pm 0.03$ & $0.39 \pm 0.01$ & $\mathrm{~N} / \mathrm{A}$ & $0.10 \pm 0.01$ & $0.19 \pm 0.01$ & $0.24 \pm 0.01$ & $0.41 \pm 0.01$ & Direct \\
\hline & $0.33 \pm 0.02$ & $0.16 \pm 0.01$ & $\mathrm{~N} / \mathrm{A}$ & $0.10 \pm 0.00$ & $0.10 \pm 0.00$ & $0.10 \pm 0.00$ & $0.09 \pm 0.00$ & GL \\
\hline & $0.28 \pm 0.01$ & $0.16 \pm 0.01$ & $\mathrm{~N} / \mathrm{A}$ & $0.03 \pm 0.00$ & $0.04 \pm 0.00$ & $0.05 \pm 0.00$ & $0.06 \pm 0.00$ & StaNoSA \\
\hline & $0.34 \pm 0.02$ & $0.15 \pm 0.01$ & $\mathrm{~N} / \mathrm{A}$ & $0.07 \pm 0.00$ & $0.08 \pm 0.00$ & $0.08 \pm 0.00$ & $0.07 \pm 0.00$ & DM \\
\hline & $0.37 \pm 0.02$ & $0.22 \pm 0.02$ & $\mathrm{~N} / \mathrm{A}$ & $0.12 \pm 0.02$ & $0.11 \pm 0.01$ & $0.15 \pm 0.01$ & $0.22 \pm 0.03$ & HS \\
\hline & $1.04 \pm 0.17$ & $0.37 \pm 0.18$ & $\mathrm{~N} / \mathrm{A}$ & $0.09 \pm 0.01$ & $0.14 \pm 0.01$ & $0.16 \pm 0.01$ & $0.23 \pm 0.02$ & MM \\
\hline & $0.38 \pm 0.00$ & $0.43 \pm 0.00$ & $\mathrm{~N} / \mathrm{A}$ & $0.32 \pm 0.01$ & $0.36 \pm 0.00$ & $0.34 \pm 0.01$ & $0.45 \pm 0.00$ & $\mathrm{RH}$ \\
\hline \multirow{7}{*}{$\downarrow \mathbf{H E}$} & $0.43 \pm 0.03$ & $0.36 \pm 0.01$ & $0.10 \pm 0.01$ & $\mathrm{~N} / \mathrm{A}$ & $0.14 \pm 0.00$ & $0.20 \pm 0.00$ & $0.39 \pm 0.01$ & Direct \\
\hline & $0.34 \pm 0.02$ & $0.16 \pm 0.01$ & $0.11 \pm 0.00$ & $\mathrm{~N} / \mathrm{A}$ & $0.09 \pm 0.00$ & $0.09 \pm 0.00$ & $0.09 \pm 0.00$ & GL \\
\hline & $0.27 \pm 0.01$ & $0.15 \pm 0.01$ & $0.06 \pm 0.00$ & $\mathrm{~N} / \mathrm{A}$ & $0.05 \pm 0.00$ & $0.05 \pm 0.00$ & $0.06 \pm 0.00$ & StaNoSA \\
\hline & $0.34 \pm 0.02$ & $0.16 \pm 0.01$ & $0.10 \pm 0.00$ & $\mathrm{~N} / \mathrm{A}$ & $0.08 \pm 0.00$ & $0.08 \pm 0.00$ & $0.07 \pm 0.00$ & DM \\
\hline & $0.39 \pm 0.02$ & $0.21 \pm 0.02$ & $0.11 \pm 0.01$ & $\mathrm{~N} / \mathrm{A}$ & $0.11 \pm 0.01$ & $0.11 \pm 0.01$ & $0.18 \pm 0.01$ & HS \\
\hline & $1.02 \pm 0.14$ & $0.35 \pm 0.17$ & $0.10 \pm 0.01$ & $\mathrm{~N} / \mathrm{A}$ & $0.12 \pm 0.00$ & $0.15 \pm 0.00$ & $0.22 \pm 0.01$ & MM \\
\hline & $0.36 \pm 0.00$ & $0.42 \pm 0.00$ & $0.29 \pm 0.00$ & $\mathrm{~N} / \mathrm{A}$ & $0.34 \pm 0.00$ & $0.32 \pm 0.00$ & $0.43 \pm 0.01$ & $\mathrm{RH}$ \\
\hline \multirow{7}{*}{$\mathbf{H} \downarrow \mathbf{E}$} & $0.45 \pm 0.03$ & $0.29 \pm 0.01$ & $0.19 \pm 0.01$ & $0.14 \pm 0.00$ & N/A & $0.11 \pm 0.00$ & $0.30 \pm 0.01$ & Direct \\
\hline & $0.37 \pm 0.02$ & $0.18 \pm 0.01$ & $0.15 \pm 0.00$ & $0.13 \pm 0.00$ & $\mathrm{~N} / \mathrm{A}$ & $0.09 \pm 0.00$ & $0.09 \pm 0.00$ & GL \\
\hline & $0.28 \pm 0.01$ & $0.15 \pm 0.01$ & $0.07 \pm 0.00$ & $0.06 \pm 0.00$ & $\mathrm{~N} / \mathrm{A}$ & $0.03 \pm 0.00$ & $0.04 \pm 0.00$ & StaNoSA \\
\hline & $0.36 \pm 0.02$ & $0.18 \pm 0.02$ & $0.15 \pm 0.00$ & $0.13 \pm 0.00$ & $\mathrm{~N} / \mathrm{A}$ & $0.08 \pm 0.00$ & $0.08 \pm 0.00$ & DM \\
\hline & $0.27 \pm 0.02$ & $0.22 \pm 0.02$ & $0.12 \pm 0.01$ & $0.11 \pm 0.01$ & $\mathrm{~N} / \mathrm{A}$ & $0.12 \pm 0.01$ & $0.20 \pm 0.02$ & HS \\
\hline & $1.25 \pm 0.14$ & $0.36 \pm 0.18$ & $0.17 \pm 0.00$ & $0.16 \pm 0.00$ & $\mathrm{~N} / \mathrm{A}$ & $0.09 \pm 0.00$ & $0.17 \pm 0.01$ & MM \\
\hline & $0.34 \pm 0.00$ & $0.36 \pm 0.00$ & $0.26 \pm 0.01$ & $0.26 \pm 0.01$ & $\mathrm{~N} / \mathrm{A}$ & $0.26 \pm 0.01$ & $0.36 \pm 0.01$ & $\mathrm{RH}$ \\
\hline \multirow{7}{*}{$\mathbf{H} \uparrow \mathbf{E}$} & $0.46 \pm 0.03$ & $0.27 \pm 0.01$ & $0.24 \pm 0.01$ & $0.20 \pm 0.00$ & $0.11 \pm 0.00$ & N/A & $0.28 \pm 0.01$ & Direct \\
\hline & $0.37 \pm 0.02$ & $0.18 \pm 0.01$ & $0.15 \pm 0.00$ & $0.13 \pm 0.00$ & $0.10 \pm 0.00$ & N/A & $0.10 \pm 0.00$ & GL \\
\hline & $0.27 \pm 0.01$ & $0.14 \pm 0.01$ & $0.07 \pm 0.00$ & $0.06 \pm 0.00$ & $0.03 \pm 0.00$ & $\mathrm{~N} / \mathrm{A}$ & $0.04 \pm 0.00$ & StaNoSA \\
\hline & $0.36 \pm 0.02$ & $0.17 \pm 0.01$ & $0.14 \pm 0.00$ & $0.12 \pm 0.00$ & $0.08 \pm 0.00$ & $\mathrm{~N} / \mathrm{A}$ & $0.08 \pm 0.00$ & $\mathrm{DM}$ \\
\hline & $0.32 \pm 0.02$ & $0.18 \pm 0.02$ & $0.14 \pm 0.01$ & $0.12 \pm 0.01$ & $0.11 \pm 0.01$ & $\mathrm{~N} / \mathrm{A}$ & $0.17 \pm 0.02$ & HS \\
\hline & $1.24 \pm 0.12$ & $0.38 \pm 0.20$ & $0.21 \pm 0.01$ & $0.19 \pm 0.00$ & $0.09 \pm 0.00$ & $\mathrm{~N} / \mathrm{A}$ & $0.17 \pm 0.01$ & MM \\
\hline & $0.31 \pm 0.00$ & $0.35 \pm 0.00$ & $0.23 \pm 0.00$ & $0.22 \pm 0.00$ & $0.26 \pm 0.00$ & $\mathrm{~N} / \mathrm{A}$ & $0.34 \pm 0.00$ & $\mathrm{RH}$ \\
\hline \multirow{7}{*}{$\uparrow \mathbf{H E}$} & $0.54 \pm 0.03$ & $0.25 \pm 0.02$ & $0.41 \pm 0.01$ & $0.39 \pm 0.01$ & $0.30 \pm 0.01$ & $0.28 \pm 0.01$ & $\mathrm{~N} / \mathrm{A}$ & Direct \\
\hline & $0.38 \pm 0.02$ & $0.20 \pm 0.02$ & $0.17 \pm 0.00$ & $0.15 \pm 0.00$ & $0.11 \pm 0.00$ & $0.12 \pm 0.00$ & $\mathrm{~N} / \mathrm{A}$ & GL \\
\hline & $0.28 \pm 0.01$ & $0.15 \pm 0.02$ & $0.09 \pm 0.00$ & $0.08 \pm 0.00$ & $0.05 \pm 0.00$ & $0.05 \pm 0.00$ & $\mathrm{~N} / \mathrm{A}$ & StaNoSA \\
\hline & $0.38 \pm 0.02$ & $0.19 \pm 0.02$ & $0.16 \pm 0.00$ & $0.14 \pm 0.00$ & $0.10 \pm 0.00$ & $0.11 \pm 0.00$ & $\mathrm{~N} / \mathrm{A}$ & DM \\
\hline & $0.27 \pm 0.02$ & $0.19 \pm 0.02$ & $0.15 \pm 0.02$ & $0.13 \pm 0.01$ & $0.14 \pm 0.01$ & $0.13 \pm 0.01$ & $\mathrm{~N} / \mathrm{A}$ & HS \\
\hline & $1.50 \pm 0.12$ & $0.44 \pm 0.26$ & $0.27 \pm 0.01$ & $0.26 \pm 0.01$ & $0.16 \pm 0.01$ & $0.16 \pm 0.01$ & $\mathrm{~N} / \mathrm{A}$ & $\mathrm{MM}$ \\
\hline & $0.29 \pm 0.00$ & $0.22 \pm 0.01$ & $0.22 \pm 0.00$ & $0.22 \pm 0.00$ & $0.18 \pm 0.00$ & $0.18 \pm 0.00$ & $\mathrm{~N} / \mathrm{A}$ & $\mathrm{RH}$ \\
\hline
\end{tabular}

Table 3: Confusion matrix showing $\mu_{p, q}$ and $\sigma_{p, q}$ across all 7 protocols of 25 images forDirect, Global Histogram (GL), Reinhard et al. (RH) 20], Macenko et al. (MM) 28, Histogram Specification (HS) 9], and Khan et al. (DM) 8]. Lowest $\mu_{p, q}$ for each group is bolded. In almost all cases, StaNoSA has the lowest $\mu_{p, q}$. 
The confusion matrix shown in Table 3 contains $\mu_{p, q}$ and $\sigma_{p, q}$ for all $p, q$. Here we stress that it is impossible for the $\mu_{p, q}$ to be zero as images are adjacent slices, not replicates as in $S_{1}$. Hence there are guaranteed to be slight differences in the visual appearance of the slides. It can be seen that StaNoSA consistently provides the smallest $\mu_{p, q}$.

In Figure 6 we present images of $S_{2}$ for qualitative evaluation, choosing specifically the most extreme of the images to normalize: $\downarrow \mathrm{H} \downarrow \mathrm{E}$ and $\uparrow \mathrm{H} \uparrow \mathrm{E}$. We can see that although the stains are notably different in $S_{2}$, our approach can successfully alter $S$ to match the color space of $T$.

\subsection{Experiment 3: Evaluate standardization via object detection}

\subsubsection{Design}

In this experiment we evaluate the effect of color standardization on a particular object detection task, namely nuclei detection. Our experiment involves selecting two H\&E images to be used as templates (Figure 7), one which is not anomalous (Figure $7 \mathrm{k}$ ) and one which is (see Figure $7 \mathrm{~b}$ ). The large proportion of red blood cells in the image tends to affect the balance of representation of tissue classes and hence can affect the performance of various color standardization algorithms. We perform color deconvolution using the H\&E stain matrix as presented in [16]. Subsequently, we identify the optimal threshold $(\psi=.914$ in this instance) on the $T$ image, by which to separate the nuclei stained pixels from other pixels in the resultant $\mathrm{H}$ channel. Lastly we normalize the 7 images of $S_{3}$ to $T$, and process them in similar fashion: (a) color deconvolution followed by (b) thresholding. To evaluate the results, we compute the Dice coefficient [31] $\left(\mu_{\phi}=\Sigma_{i} \phi(i) / N, i \in\{i, \ldots, N\}\right)$ and its variance $\left(\sigma_{\phi}\right)$ of the resulting nuclei as compared to the manually annotated ground truth for all approaches (e.g., Direct, GL, StaNoSA, DM, HS, MM, and RH).

We chose an H\&E image from $S_{2}$ to act as $T$, but one in particular which does not have balanced class proportions (Figure $7 \mathrm{~b}$ ). We specifically selected the template image in Figure $7 \mathrm{~b}$ to determine if the methods we compare against (Direct, GL, StaNoSA, DM, HS, MM, and RH) are robust against imbalanced 
tissue class representations. To provide a comparison, $T$ shown in Figure 7 a does not have any unusual artifacts which disrupt the relative class proportionality. 4.6.2. Results

As we can see from Figure 8 and Figure 9 the StaNoSA is able to improve $\mu_{\phi}$ by $10 \%$ while reducing $\sigma_{\phi}$. One of the difficulties with color deconvolution, as discussed in [8], is the fact that the approach needs careful selection of the appropriate stain matrix in order to achieve satisfactory results. In this case, because we have seven different staining protocols, it is unlikely that the same matrix would consistently work well. Instead, using StaNoSA, we find $T$ which works well and then shift $S_{3}$ to that image.

In the cases of $\uparrow \mathrm{H}$ and $\uparrow \mathrm{H} \uparrow \mathrm{E}$ we see significant improvements in color constantcy across $S_{3}$ as a result of StaNoSA. As expected, GL and HS (global normalization techniques) perform poorly because of the large red blood cell artifacts. On the other hand, when these artifacts are not present, the GL, HS, and StaNoSA normalization technique perform comparably.

\section{Discussion}

\section{Color standardization of digital histopathology images is critical to reducing} stain variability and improving the robustness of computer assisted diagnostic and image quantification algorithms such as nuclei and mitoses detection. Previous approaches have potentially been handicapped by the necessity of accurately defining a stain matrix or requiring images to have similar tissue type representations in the image (i.e., similar proportions of stroma, nuclei). StaNoSA is able to circumvent the need for a stain matrix by identifying tissue sub-types within the image in an unsupervised manner. The large number of tissue sub-types produced affords the opportunity of managing class imbalances better than previous approaches, by providing greater specificity through more tightly defined clusters. In this manner, only very similar pixels in the template image are used to normalize a target pixel, as opposed to all pixels at a histologic primitive level (e.g., all epithelial pixels). The larger number of clusters helps manage 
situations where there is a significant proportional difference in the presence of individual tissue classes in the target and template images.

Traditionally, the most important aspect of a normalization process is choosing an optimal template image. While it makes sense to develop algorithms with as much robustness as possible, inferring cohort parameters from a poor template image will typically be a challenging proposition. The most important quality associated with a good template image, especially in the context of color standardization approaches, is that it should be representative of the other cases within the cohort. Theoretically one would ideally wish to have a correspondence from every pixel value (or color) within the template image to a corresponding pixel color in each of the cohort images, so that the color distributions can be brought into alignment. Since having such an idealized template image is highly unlikely, we attempt to select one which comes as close as possible to such an ideal image. This therefore implies the need for template images which proprtionately cover the various tissue classes of interest - nuclei, stroma, epithelium, blood vessels, etc. The presence of these tissue classes are imperative within the target and template images in order to be able to identify and align tissue clusters.

In general, normalization of images becomes increasingly difficult when the variations in staining of the slides increases. Our results show that in many cases, when the moving and template images are similar in terms of their staining characteristics, less sophistocated approaches tend to do very well. It is in scenarios where there is large discrepancy in staining variations between slides that the StaNoSA approach is able to demonstrate a substantial improvement over the state of the art. As StaNoSA is computationally more burdensome, it may be possible to selectively invoke StaNoSA in only those settings where it is determined that the tissue class representation for the target and template images differ substantially.

\section{Concluding Remarks}


In this work, we present a new color standardization approach called Stain Normalization using Sparse AutoEncoders (StaNoSA) which attempts to address the limitations of previous related approaches. We leverage the intuition that filters learned via deep learning tend to respond similarly to tissue subtypes having similar characteristics, even across images. This invariance allows for accurate, and unsupervised, partitioning of the tissue compartments for subsequent histogram matching and alignment. In a comparison of StaNoSA with 4 different color standardization approaches (one of them being a recent state of the art scheme), StaNoSA was able to (1) reduce inter scanner color variance to within the range observed in images scanned multiple times on the same scanner, (2) reduce extreme variations in color induced by differential staining, slide preparation, and slide digitization, and (3) improve performance of subsequent image processing algorithms, specifically nuclei detection. In a majority of cases, StaNoSA outperformed the other comparative approaches, in many cases by over $50 \%$. In conclusion, StaNoSA appears to be able to handle situations wherein tissue classes are disproportionately represented between target and template images. Future work will entail a more rigorous and automated way of identifying the algorithmic parameters (e.g. number of clusters $K$ ) and larger scale validation studies.

\section{Acknowledgments}

Research reported in this publication was supported by the National Cancer Institute of the National Institutes of Health under award numbers 1U24CA19937401, R21CA167811-01, R21CA179327-01; R21CA195152-01 the National Institute of Diabetes and Digestive and Kidney Diseases under award number R01DK09850302, the DOD Prostate Cancer Synergistic Idea Development Award (PC120857); the DOD Lung Cancer Idea Development New Investigator Award (LC130463), the DOD Prostate Cancer Idea Development Award; the Ohio Third Frontier Technology development Grant, the CTSC Coulter Annual Pilot Grant, the Case Comprehensive Cancer Center Pilot Grant VelaSano Grant from the 
Cleveland Clinic the Wallace H. Coulter Foundation Program in the Department of Biomedical Engineering at Case Western Reserve University.

The content is solely the responsibility of the authors and does not necessarily represent the official views of the National Institutes of Health.

\section{References}

[1] M. Gurcan, L. Boucheron, et al., Histopathological image analysis: a review., IEEE Rev Biomed Eng 2 (2009) 147-171.

[2] M. Veta, J. Pluim, et al., Breast cancer histopathology image analysis: a review., IEEE Trans Biomed Eng 61 (5) (2014) 1400-1411.

[3] J. Monaco, J. Hipp, et al., Image segmentation with implicit color standardization using spatially constrained expectation maximization: detection of nuclei., International Conference on Medical Image Computing and Computer-Assisted Intervention (MICCAI) 15 (2012) 365-72.

[4] A. M. Khan, H. El-Daly, N. Rajpoot, Ranpec: Random projections with ensemble clustering for segmentation of tumor areas in breast histology images, in: Medical Image Understanding and Analysis (MIUA), British Machine Vision Association (BMVA), 2012, pp. 17-23.

[5] J. Hipp, J. Cheng, et al., Spatially invariant vector quantization: A pattern matching algorithm for multiple classes of image subject matter including pathology., J Pathol Inform 2 (2011) 13.

[6] J. Kong, O. Sertel, et al., Computer-aided grading of neuroblastic differentiation: Multi-resolution and multi-classifier approach., in: ICIP (5), IEEE, 2007, pp. 525-528.

[7] Y. Wang, S. Chang, et al., A color-based approach for automated segmentation in tumor tissue classification., Conf Proc IEEE Eng Med Biol Soc 2007 (2007) 6577-6580. 
[8] A. Khan, N. Rajpoot, et al., A nonlinear mapping approach to stain normalization in digital histopathology images using image-specific color deconvolution., IEEE Trans Biomed Eng 61 (6) (2014) 1729-1738.

[9] A. K. Jain, Fundamentals of Digital Image Processing, Prentice-Hall, Inc., Upper Saddle River, NJ, USA, 1989.

[10] H. Irshad, A. Veillard, et al., Methods for nuclei detection, segmentation, and classification in digital histopathology: A review - current status and future potential, Biomedical Engineering, IEEE Reviews in 7 (2014) 97114.

[11] A. Sahirzeeshan, A. Madabhushi, An integrated region-, boundary, shapebased active contour for multiple object overlap resolution in histological imagery., IEEE transactions on medical imaging 31 (2012) 1448-60.

[12] H. Fatakdawala, J. Xu, et al., Expectation-maximization-driven geodesic active contour with overlap resolution (emagacor): application to lymphocyte segmentation on breast cancer histopathology., IEEE Transactions on Biomedical Engineering 57 (7) (2010) 1676-1689.

[13] J. Xu, L. Xiang, et al., Stacked sparse autoencoder (ssae) for nuclei detection on breast cancer histopathology images., IEEE Transactions on Medical Imaging.

[14] F. Tian, B. Gao, et al., Learning deep representations for graph clustering, in: Proceedings of the Twenty-Eighth AAAI Conference on Artificial Intelligence, July 27 -31, 2014, Québec City, Québec, Canada., 2014, pp. 1293-1299.

[15] P. Vincent, H. Larochelle, et al., Extracting and composing robust features with denoising autoencoders, in: Proceedings of the 25th International Conference on Machine Learning, ICML '08, ACM, New York, NY, USA, 2008, pp. 1096-1103. 
[16] A. C. Ruifrok, D. A. Johnston, Quantification of histochemical staining by color deconvolution., Anal Quant Cytol Histol 23 (4) (2001) 291-299.

[17] P. W. Hamilton, P. H. Bartels, et al., Automated location of dysplastic fields in colorectal histology using image texture analysis., J Pathol 182 (1) (1997) 68-75.

[18] H. Qureshi, O. Sertel, et al., Adaptive discriminant wavelet packet transform and local binary patterns for meningioma subtype classification., Med Image Comput Comput Assist Interv 11 (Pt 2) (2008) 196-204.

[19] A. Ruiz, O. Sertel, et al., Pathological image analysis using the gpu: Stroma classification for neuroblastoma, in: Bioinformatics and Biomedicine, 2007. BIBM 2007. IEEE International Conference on, 2007, pp. 78-88.

[20] E. Reinhard, M. Ashikhmin, et al., Color transfer between images, IEEE Comput. Graph. Appl. 21 (5) (2001) 34-41.

[21] D. Magee, D. Treanor, et al., Colour normalisation in digital histopathology images, in: Proc. Optical Tissue Image analysis in Microscopy, Histopathology and Endoscopy (MICCAI Workshop), 2009, pp. 100-111.

[22] A. Rabinovich, S. Agarwal, et al., Unsupervised color decomposition of histologically stained tissue samples, in: S. Thrun, L. Saul, B. Schölkopf (Eds.), Advances in Neural Information Processing Systems 16, MIT Press, 2004, pp. 667-674.

[23] A. Basavanhally, A. Madabhushi, Em-based segmentation-driven color standardization of digitized histopathology, in: Proc. SPIE, Vol. 8676, 2013, pp. $86760 \mathrm{G}-86760 \mathrm{G}-12$.

[24] Y. Bengio, P. Lamblin, et al., Greedy layer-wise training of deep networks, in: In NIPS, MIT Press, 2007.

[25] Y. Bengio, Learning deep architectures for ai, Foundations and Trends in Machine Learning 2 (1) (2009) 1-127. 
[26] T. Zhang, Solving large scale linear prediction problems using stochastic gradient descent algorithms, in: ICML 2004: Proceedings of the 21st International Conference on Machine Learning, 2004, pp. 919-926.

[27] A. Coates, H. Lee, A. Ng, An analysis of single-layer networks in unsupervised feature learning, in: G. Gordon, D. Dunson, M. Dudk (Eds.), Proceedings of the Fourteenth International Conference on Artificial Intelligence and Statistics, Vol. 15 of JMLR Workshop and Conference Proceedings, 2011, pp. 215-223.

[28] M. Macenko, M. Niethammer, et al., A method for normalizing histology slides for quantitative analysis, in: Biomedical Imaging: From Nano to Macro, 2009. ISBI '09. IEEE International Symposium on, 2009, pp. 11071110.

[29] I. J. Goodfellow, D. Warde-Farley, P. Lamblin, V. Dumoulin, M. Mirza, R. Pascanu, J. Bergstra, F. Bastien, Y. Bengio, Pylearn2: a machine learning research library, arXiv preprint arXiv:1308.4214.

[30] J. Bergstra, O. Breuleux, et al., Theano: a CPU and GPU math expression compiler, in: Proceedings of the Python for Scientific Computing Conference (SciPy), 2010, oral Presentation.

[31] L. Dice, Measures of the amount of ecologic association between species, Ecology 26 (3) (1945) 297-302. 


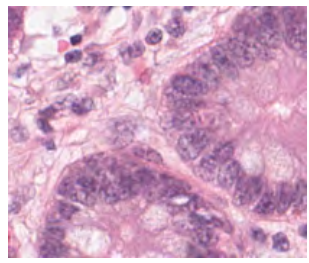

(a)

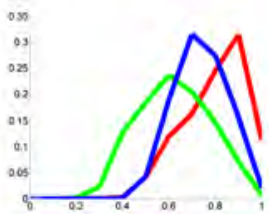

(d)

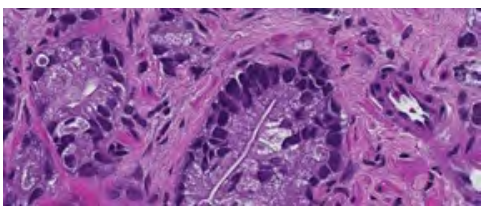

(g)

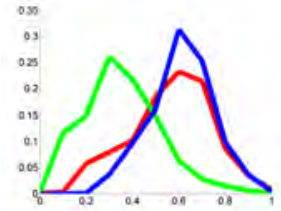

(i)

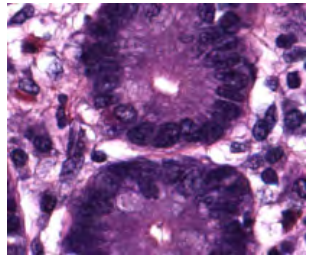

(b)

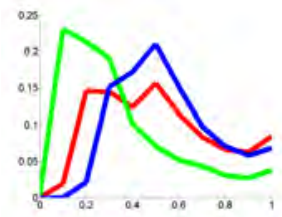

(e)

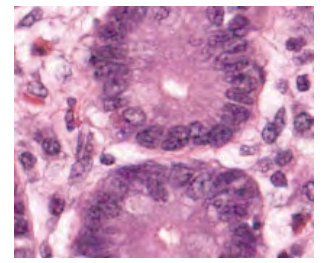

(c)

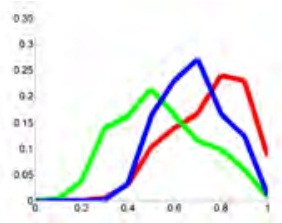

(f)

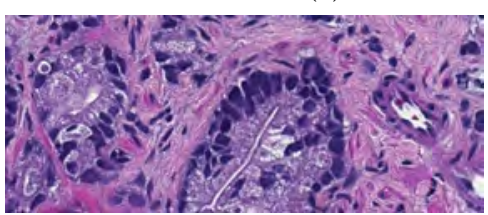

(h)

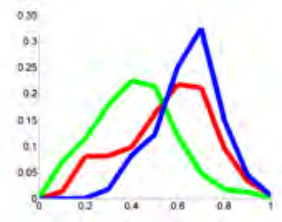

(j)

Figure 1: Visual appearance of the H\&E slides can differ greatly depending on protocols and equipment used for staining. A GI stained H\&E sample (a) acts as a template image, while the target image (b) is another H\&E GI image in which we can see a strong visual difference due to staining protocol. Finally, after applying StaNoSA we can see (c) a shifted version of (b) which is in the same color space as (a). Their respective intensity distributions for each of the R, G, B color channels are presented in panels (d)-(f) respectively, which in turn show that the color distributions for the target images histogram (e) is heavily skewed towards the left. After applying StaNoSA (f) the intensity distribution for each of the 3 channels begins to more closely resemble that of the template image (d). Panels (g) and (h) show the same tissue slide but digitized with a (g) Leica scanner and (h) Ventana scanner, respectively. We can see that even though the only difference between the two images is the fact that they were digitized on two different scanners, panel (g) reveals more of a pink hue while (h) presents with a darker purple appearance. These differences are reflected in the corresponding color distributions $((\mathrm{i}),(\mathrm{j}))$ for the two images in $(\mathrm{g})$ and $(\mathrm{h})$ respectively. 
Figure 2: An illustrative flowchart showing the StaNoSA process of color standardization. 


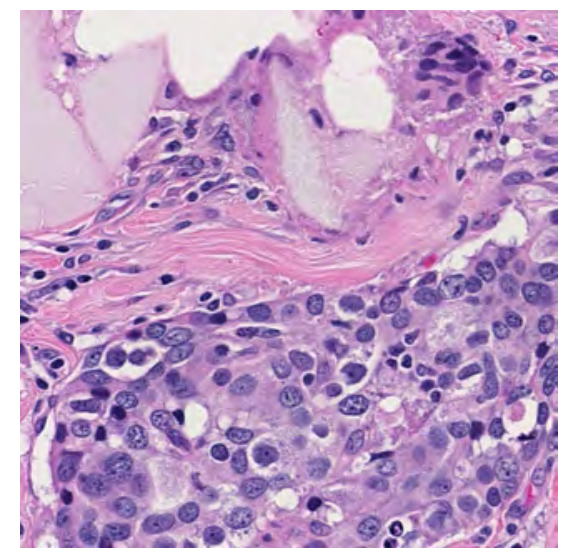

(a)

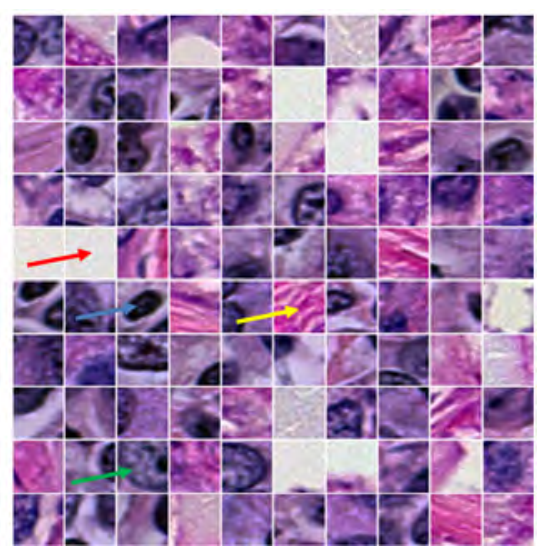

(b)

Figure 3: Original image (a) used as $\mathcal{T}$ with a sampling of (b) random training patches. For a sparse autoencoder to learn representative filters well, we need to ensure an image is well represented from the patches which are extracted. We can see in (b) that our sampling procedure provides a nice distribution of the various classes which are present in the image such as background (red arrow), nuclei (green arrow), lymphocytes (blue arrow), and stroma regions (yellow arrow). 


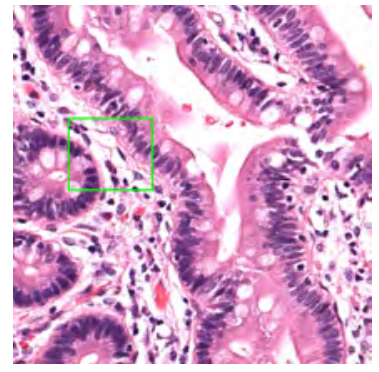

(a)

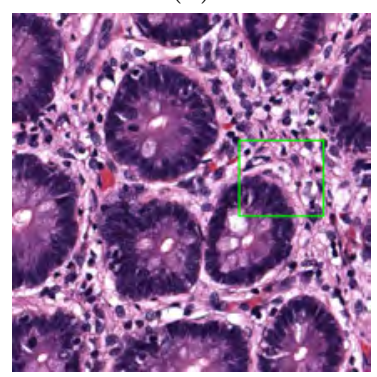

(d)

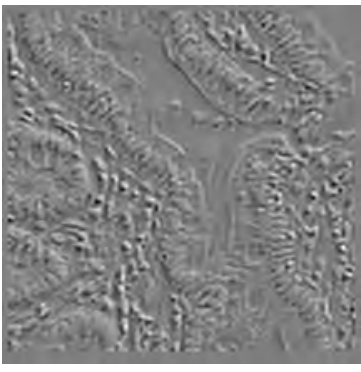

(b)

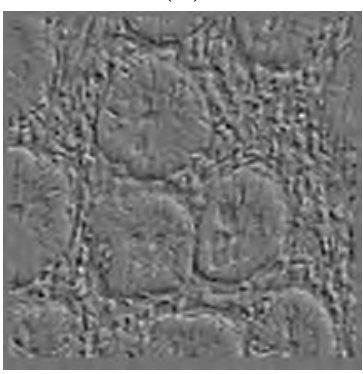

(e)

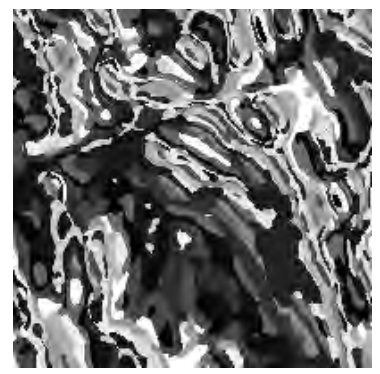

(c)

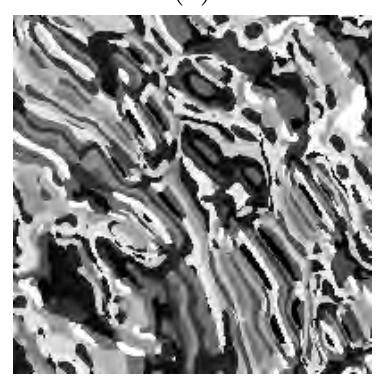

(f)

Figure 4: Illustration of different filter responses via the StaNoSA algorithm on H\&E images. We can see that both the (a) template image and (d) moving image respond to an arbitrarily selected (b) \& (e) filter in similar ways. Extracted from the green boxes of (a) and (d), the assignment of each pixel to one of 50 clusters can be viewed in gray scale shown in (c) and (f), respectively. 


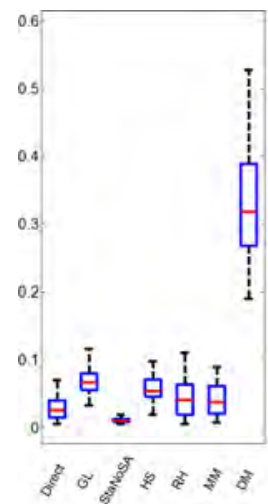

(a)

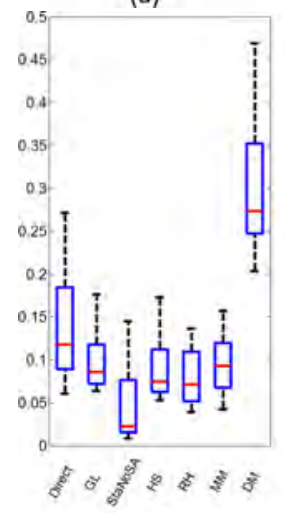

(d)

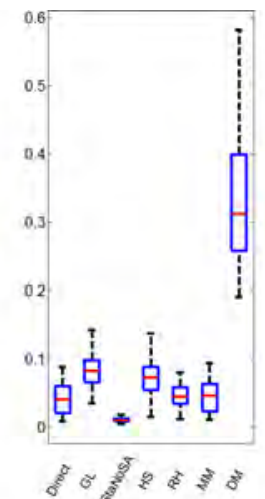

(b)

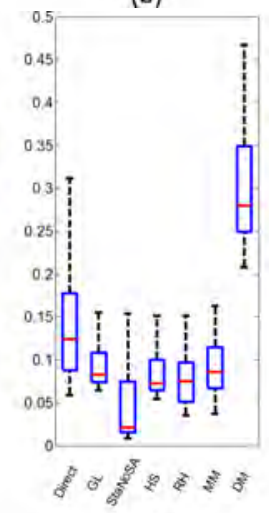

(e)

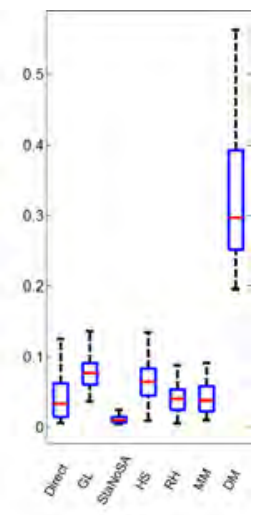

(c)

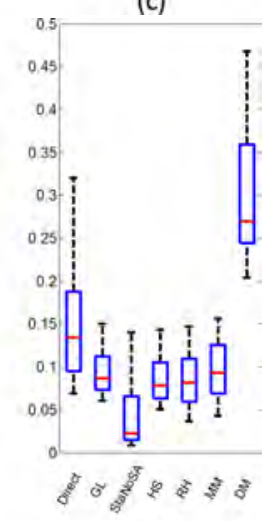

(f)

Figure 5: Box and Whisker plots showing $\mu^{i n}$ and $\sigma^{i n}$ for intra scanner differences ((a)-(c)). We compare (a) $S_{1, V_{1}}$ to $S_{1, V_{2}}$, and (b) $S_{1, V_{3}}$ and then (c) $S_{1, V_{2}}$ to $S_{1, V_{3}}$. Red line indicates $\mu^{i n}$, the blue box bounds the 25 th percentile and the black whiskers extend to the 75 th percentile. It can be seen quite clearly that our StaNoSA approach not only reduces $\mu^{i n}$, but also reduce variance across $\sigma^{i n}$. Additionally, we compare ((d)-(f)) $S_{1, L}$ to $S_{1, V_{i}} i \in\{1,2,3\}$ and see a similar trend for $\mu^{i t}$ and $\sigma^{i t}$. 

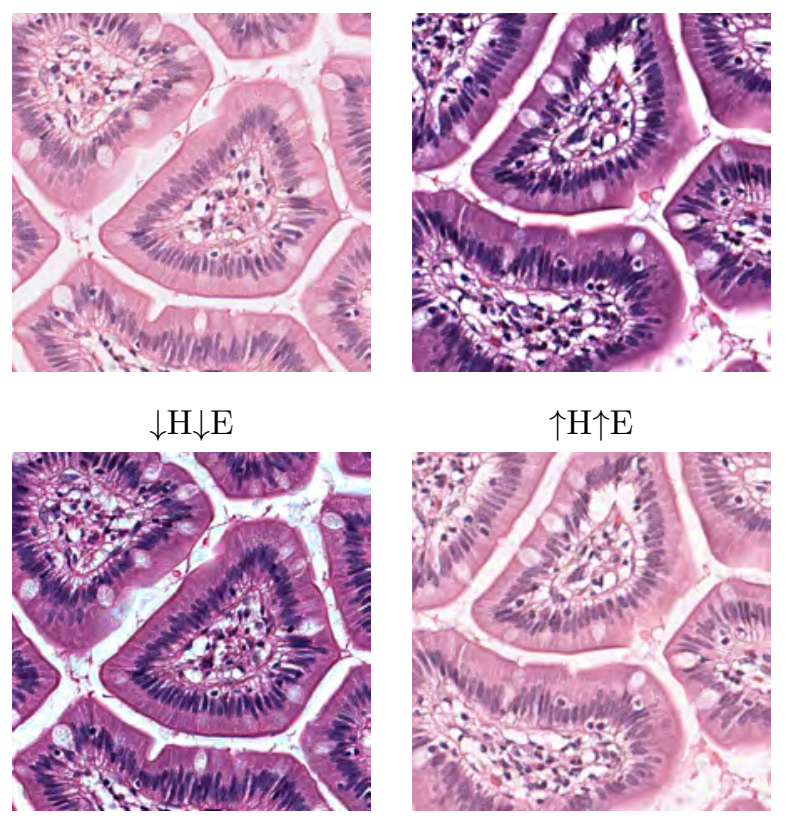

$\downarrow \mathrm{H} \downarrow \mathrm{E}$ in $\uparrow \mathrm{H} \uparrow \mathrm{E}$ color
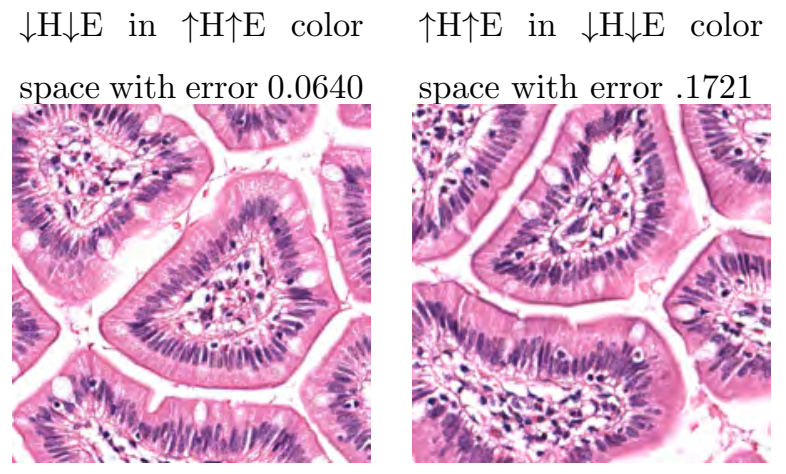

$\downarrow \mathrm{H} \downarrow \mathrm{E} \quad$ in $\mathrm{HE} \quad$ color

$\uparrow \mathrm{H} \uparrow \mathrm{E}$ in $\mathrm{HE}$ color

space with error 0.0524

space with error 0.0249

Figure 6: The original $\downarrow \mathrm{H} \downarrow \mathrm{E}$ and $\uparrow \mathrm{H} \uparrow \mathrm{E}$ images in the top row. In the middle row we normalize to the others color space, such that $\uparrow \mathrm{H} \uparrow \mathrm{E}$ gets normalized to $\downarrow \mathrm{H} \downarrow \mathrm{E}$ and $\downarrow \mathrm{H} \downarrow \mathrm{E}$ gets normalized to $\uparrow \mathrm{H} \uparrow \mathrm{E}$. Lastly, we show both of them being normalized to the standard HE image. In all cases, we can see that the images strongly resemble their template image color characteristics. 


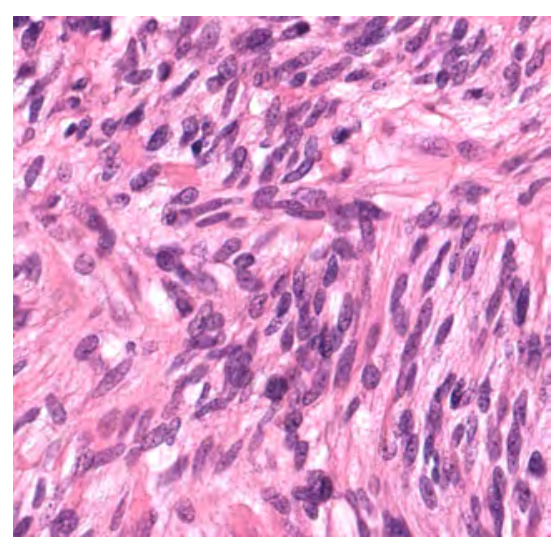

(a)

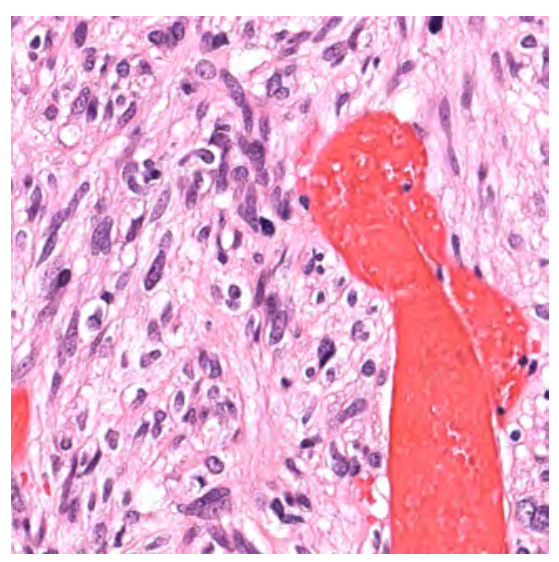

(b)

Figure 7: Typical (a) $T$ versus a (b) $T$ used which was specifically chosen because of the class dis-proportionality. In particular we see the large pink object, of red blood cells in (b), on the right side of the screen. It would be expected that this affects the GL and HS approachs as non-red blood cells in $S$ are aligned with the red blood cells histogram components. 


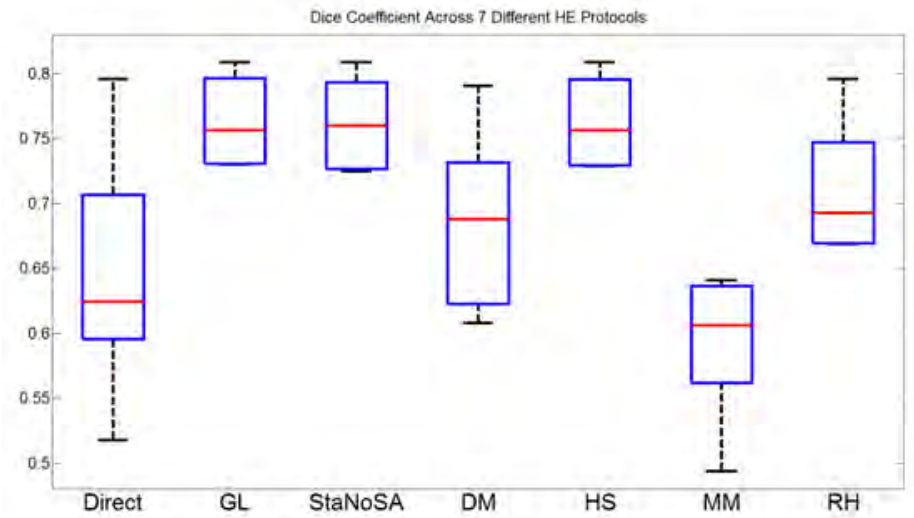

(a)

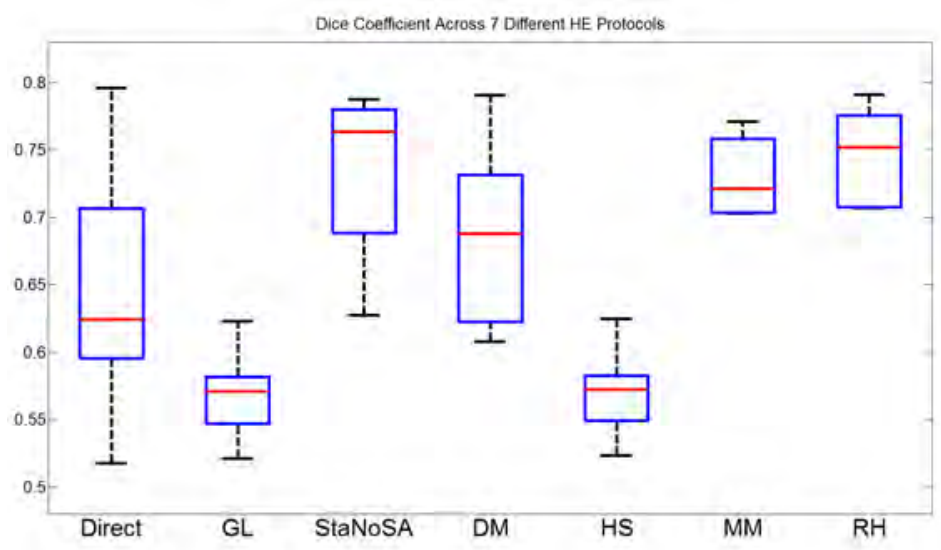

(b)

Figure 8: Box and Whisker plots showing $\mu_{\phi}$ and $\sigma_{\phi}$ before normalization and normalization using Direct, Global Histogram (GL), Reinhard et al. (RH) [20, Macenko et al. (MM) 28], Histogram Specification (HS) [9], and Khan et al. (DM) 8]. Panel (a) shows the instances where the template and moving image share similar tissue class proportions, while (b) illustrates the consequences of having imbalanced class proportions. The Red line indicates the mean, the blue box bounds the 25th percentile and the black whiskers extend to the 75 th percentile. 


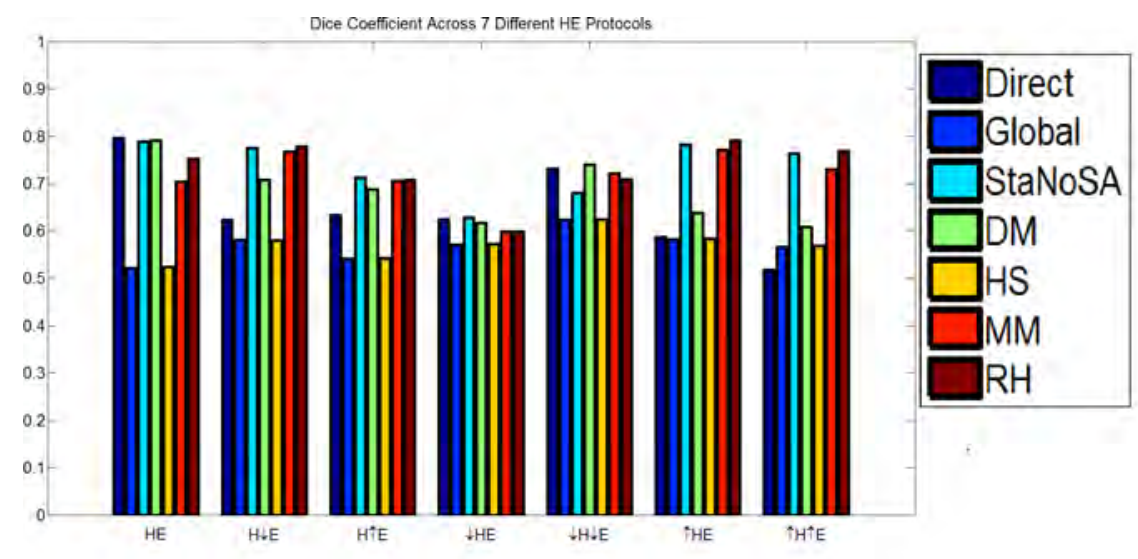

Figure 9: Bar plots showing $\mu_{\phi}$ on a per staining protocol basis ( $\mathrm{HE}, \mathrm{H} \downarrow \mathrm{E}, \mathrm{H} \uparrow \mathrm{E}, \downarrow \mathrm{HE}, \downarrow \mathrm{H} \downarrow \mathrm{E}$, $\uparrow \mathrm{HE}$, and $\uparrow \mathrm{H} \uparrow \mathrm{E}$, where $\uparrow$ and $\downarrow$ indicate over- and under-staining of the specified dye) before normalization and after normalization using Direct, Global Histogram, Reinhard et al. (RH) [20, Macenko et al. (MM) 28, Histogram Specification (HS) 9], and Khan et al. (DM) 8] 